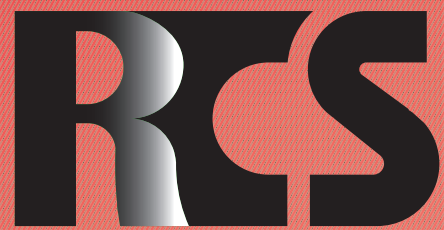

Depósito legal ppi $201502 Z U 4662$

Esta publicación científica en formato digital es continuidad de la revista impresa Depósito Legal: pp $197402 Z$ Z789

- ISSN: 1315-9518 • ISSN-E: 2477-9431

Universidad del Zulia. Revista de la Facultad de Ciencias Económicas y Sociales Vol. XXVI. No.1 


\title{
Impacto de las transferencias federales en la deuda municipal de los ayuntamientos mexicanos
}

\author{
Herrera Díaz de León, Luis Lenin* \\ González Acolt, Roberto** \\ Alcantar López, Cristian Omar ${ }^{* * *}$ \\ Govea Franco, Ángeles Montserrat ${ }^{* * * *}$
}

\section{Resumen}

Con las reformas llevadas a cabo en la Ley de coordinación fiscal en el año 2007 se incrementaron los recursos transferidos a los municipios mexicanos, los cuales ayudarían a mejorar aspectos de las finanzas públicas de los ayuntamientos, uno de ellos el nivel de obligaciones con terceros. El presente artículo tiene como objetivo identificar la correlación que hay entre los recursos que envía la federación y el comportamiento de sus deudas. Se hizo un análisis de corte longitudinal por medio de la técnica econométrica de Datos de Panel a los 44 municipios de México más grandes en población, durante los años 2000 a 2016; además de usar una variable de control dicotómica que es el factor político. Entre los resultados se logra demostrar una relación positiva entre el pago de deuda y las transferencias con un nivel de significancia al $10 \%$ donde por cada mil pesos de incrementos en transferencias por habitantes, la deuda per cápita se incrementa en 86,49 pesos mexicanos en este periodo de tiempo y no se encuentra evidencia de que el factor político influya en la variable dependiente. Se concluye, que queda demostrado el incentivo a endeudarse impulsado por el envío de mayores recursos federales.

Palabras clave: Hacienda pública; política fiscal; transferencias federales; deuda municipal; ayuntamientos mexicanos.

\footnotetext{
Doctor en Estudios Fiscales con Orientación en Hacienda Pública. Maestro en Comercio Internacional. Profesor de Tiempo Completo de la Universidad Autónoma de Aguascalientes, México. Perfil PRODEP. E-mail: lenincpf@yahoo.com.mx (iD) ORCID: https://orcid.org/0000-0002-9784-9275

** Doctor en Ciencias con Orientación en Economía. Profesor de Tiempo Completo de la Universidad Autónoma de Aguascalientes, México. Miembro del Sistema Nacional de Investigación nivel 1. Perfil PRODEP. E-mail: rgonza@correo.uaa.mx (iD ORCID: https://orcid.org/0000-0001-5468-3028

*** Doctor en Estudios Fiscales con Orientación en Hacienda Pública. Profesor de Tiempo Completo de la Universidad de Guadalajara, México. Miembro del Sistema Nacional de Investigación nivel "C". Profesor con perfil PRODEP. E-mail: cristian_alcantar@hotmail.com iD ORCID: https://orcid. org/0000-0002-1436-6711

**** Estudiante de Doctorado en Ciencias Administrativas en la Universidad Autónoma de Aguascalientes, Mexico. E-mail: angeles.govea@edu.uaa.mx (iD) ORCID: https://orcid.org/0000-0002-0949-2705
}

Recibido: 2020-09-15 · Aceptado: 2020-12-03 


\title{
Impact of federal transfers on the municipal debt of Mexican Municipalities
}

\begin{abstract}
With the reforms carried out in the Fiscal Coordination Law in 2007, the resources transferred to Mexican municipalities were increased, which would help to improve aspects of the public finances of the municipalities, one of them the level of obligations with third parties. The objective of this article is to identify the correlation between the resources sent by the federation and the behavior of its debts. A longitudinal section analysis was carried out using the Panel Data econometric technique to the 44 largest municipalities in Mexico in population, during the years 2000 to 2016; in addition to using a dichotomous control variable that is the political factor. Among the results, it is possible to demonstrate a positive relationship between the payment of debt and transfers with a significance level of $10 \%$ where for every thousand pesos of increases in transfers per inhabitant, the debt per capita increases by 86.49 Mexican pesos in this period of time and there is no evidence that the political factor influences the dependent variable. It is concluded that the incentive to get into debt driven by the sending of greater federal resources is demonstrated.
\end{abstract}

Keywords: Public estate; fiscal policy; federal transfers; municipal debt; Mexican municipalities.

\section{Introducción}

El federalismo fiscal, de acuerdo con Oates (1972), estudia cómo se constituyen las relaciones y características de los atributos y las responsabilidades en los diferentes órdenes o niveles de gobierno dentro de un Estado federal. Estas correspondencias de facultades y obligaciones determinan la forma en que se estructuran las políticas de ingresos así como egresos en un gobierno de tipo federal para poder hacer frente a los mandatos constitucionales a que están comprometidos, cuyas manifestaciones se plasman en un pacto fiscal federal.

En México, el último gran cambio de su pacto fiscal entre diferentes niveles de gobierno se dio cuando Felipe Calderón llevó a cabo una serie de reformas a las leyes de coordinación fiscal en el año 2007, con la finalidad de reforzar las finanzas públicas de los gobiernos subnacionales y dotarles de recursos para poder cumplir con sus obligaciones constitucionales en materia de gasto público. Este ultimo de acuerdo con Machado (2017) se refiere al "total del conjunto de erogaciones pautado en la Ley de Presupuesto de un determinado año, sumado a las modificaciones presupuestarias que se realizan durante el transcurso de dicho año" (p.103).

En el caso de los municipios, el principal beneficio va enfocado a una mayor cantidad de recursos transferidos para ser aplicados en sus respectivas demarcaciones, siendo algunos de ellos no etiquetados y otros con una asignación específica. Dentro de los objetivos perseguidos en el federalismo fiscal mexicano está el de ayudar a estabilizar las finanzas locales de los ayuntamientos y con esto poder disminuir desequilibrios entre los ingresos disponibles y las necesidades de gasto público para atender las demandas de sus habitantes.

El resultado es que los gobiernos subnacionales habían estado cubriendo éste faltante con deuda, lo que a largo plazo generaba problemas para las arcas municipales; por ello suministrarles más recursos debe generar una menor supeditación a este tipo de financiamiento (Secretaría de Gobernación [SEGOB], 2008). Sin embargo, 
la prominente contribución de los recursos centrales en la disponibilidad de ingresos de las entidades federales provoca una dependencia que restringe el potencial para atender los problemas locales (Chiguil, 2014).

Cuando hay déficits fiscales en cualquier nivel de gobierno, estos son cubiertos con préstamos cuyos derechos están en manos de terceros (Stiglitz y Rosengard, 2016), por lo que si su destino no es en bienes de capital o se disminuye en el futuro con superávits, en un cierto tiempo determinado impactará en la capacidad de atender necesidades regionales por el hecho de tener comprometidos los ingresos venideros. Al respecto, sostienen Prats y Rocamora (2018) que "el control del déficit debiera basarse en restricciones del endeudamiento, combinado con reducciones del nivel de gasto público" (p.15). Sobre la restricción presupuestaria, Stiglitz y Rosengard (2016) indica que si un gobierno incrementa una de sus fuentes de ingresos, sus opciones son disminuir otras contribuciones a sus ciudadanos, incrementar el gasto o disminuir la deuda (o combinaciones entre ellas).

Estudios recientes indican que hay mayores índices de deuda en los gobiernos estatales cuando se carece de legislación y reglamentación para transparentar el uso de la deuda, en contraste con aquellas entidades federativas en las que el marco legal impone límites a las cantidades a endeudarse (Astudillo, Blancas y Fonseca, 2017); lo cual refuerza los argumentos en torno a la falta de controles existentes en los gobiernos subnacionales de segundo nivel, que coadyuvan a un manejo irresponsable de ciertas fuentes de financiamiento como en este caso el de los adeudos.

Ruiz-Porras y García-Vázquez (2014), hacen planteamientos en los que se evalúa la tesis de que "son criterios económicos y políticos los que estimulan tendencias a la hora de diseñar la forma en que las transferencias son asignadas en los municipios de Jalisco" (p.79). Otros trabajos enfocan sus esfuerzos en demostrar los efectos que tiene la deuda en otras variables para el caso de gobiernos en México. De esta manera, se encuentran casos que van desde la falta de información sobre el destino final de los recursos adquiridos con débito a pesar de poder conocer los montos de estos (Astudillo y Porras, 2018), hasta analizar las correlaciones entre deuda e inversión en capital por parte de los gobiernos, encontrándose muy baja relación, siendo incluso negativa por las presiones que genera al gasto público, los compromisos futuros adquiridos en el presente (García, 2016).

Este artículo presenta un análisis de la relación existente entre las transferencias federales a los 44 municipios más grandes del país en población y el comportamiento de los pagos de deuda de cada uno de ellos. El objetivo principal de este trabajo de investigación es encontrar una correlación negativa entre los recursos federales que envía el gobierno central mexicano a los gobiernos más grandes del país a nivel municipal y los montos de pago de deuda que erogan. Con ello se pretende demostrar que mientras más recursos envía la federación a los gobiernos subnacionales de tercer nivel jerárquico mayor deuda van adquiriendo estos.

Esta situación contradice lo establecido por los objetivos de la reforma llevada a cabo en el año 2007 en lo relativo a dotar de más recursos para cubrir las necesidades de gasto de los municipios y por ende tener menos necesidad de endeudarse, es decir, que tener incrementos en participaciones y aportaciones federales debería de impactar en reducciones de los pagos de deuda.

El estudio abarca los años 2000 a 2016 y a su vez se subdivide en dos periodos: Uno, antes de la reforma de 2007; y otro, posterior a dicho evento crítico. El primer bloque temporal, comprende desde el año 2000 al año 2007; y el segundo, de los años 2008 a 2016; con la finalidad de ver los comportamientos de las variables estudiadas antes y después de los cambios en las reglas de operación para transferir recursos federales. Esta división obedece a que se quiere demostrar si con la reforma a las reglas de operación de la Ley de coordinación fiscal llevada a cabo en el año 2007, hubo cambios respecto al periodo previo a dichos arreglos legales e identificar 
Herrera Díaz de León, Luis Lenin; González Acolt, Roberto; Alcantar López, Cristian Omar y Govea Franco, Ángeles Montserrat

Impacto de las transferencias federales en la deuda municipal de los ayuntamientos mexicanos

la correlación que hay entre los recursos que envía la federación y el comportamiento de sus deudas.

El resto de la investigación se presenta en tres partes: La primera, explica con detalle la metodología empleada para el análisis econométrico llevado a cabo; la segunda parte, presenta los resultados de la investigación; y la tercera, muestra las conclusiones del autor respecto a los hallazgos encontrados en el apartado anterior.

\section{Fundamentación teórica}

\subsection{Federalismo fiscal un medio para las relaciones intergubernamentales en materia fiscal}

Las relaciones que se presentan en distintos niveles de gobierno dentro de una federación, se centran en dos grandes esferas: La estructura constitucional y el federalismo fiscal. La primera, se enfoca en el respeto y acatamiento de las leyes que emanan de la carta magna de un país; mientras que la segunda, lo hace en la participación de las diferentes jerarquías de la administración pública en el diseño de ingresos y gasto público. El presente trabajo se desarrolla dentro de los estudios de la Hacienda Pública en la rama del Federalismo Fiscal; a continuación, se presentan algunas definiciones que se emplean en la literatura académica.

El federalismo fiscal, es un conjunto de normas relativas a la recaudación de impuestos y el gasto público, en el que se da definición de competencias en una administración federal entre diferentes niveles de gobierno (Pliego, 2010). Este mismo autor resalta que en el caso mexicano ha sido un proceso histórico que ha devengado en una centralización del poder político y económico en un modelo federal.

García (2016), ve en el federalismo fiscal una estructura sistemática que asigna facultades y competencias, tanto en ingresos como en egresos públicos en los diferentes niveles de gobierno y cuyo objeto de estudio en su vertiente económica son las relaciones que se dan entre estos para la asignación y distribución de recursos. Se hace crítica sobre la fuerte centralización de esta doctrina que ha acabado fortaleciendo al gobierno federal y su titular de quien se esperan soluciones. Otra definición es: "El arreglo espacial del poder reconocido y garantizado por la Constitución Política y expresado a través de las finanzas públicas y como función pública representativa del poder político" (Mandujano, 2011, p.21).

De esta manera, tomando los elementos comunes a los autores citados, para el presente trabajo el Federalismo Fiscal se entiende como una institución rectora de las relaciones intergubernamentales en materia fiscal, en las que se asignan responsabilidades y potestades nacidas en ley, y por ende de carácter obligatorio, pero con ciertos márgenes de autonomía para el logro de objetivos económicos que impactan en los gobernados. La materialización de esas relaciones, son las transferencias federales que hace el gobierno central hacía los gobiernos subnacionales de acuerdo con los mandatos constitucionales y legales establecidos en la ley de coordinación fiscal para el caso mexicano o su legislación equivalente en otros países.

\subsection{Las transferencias federales para el bienestar social}

Las transferencias federales, son recursos financieros que son otorgados a los dos niveles de gobierno inferiores por parte del gobierno central - federal, como parte de los acuerdos que se derivan de pactos fiscales, para coadyuvar a los primeros en el cumplimiento de sus obligaciones constitucionales en favor de sus habitantes.

Los estudios teóricos relativos al federalismo fiscal armonizan elementos de economía, así como de materia política y se reconocen en el aspecto académico dos generaciones de estudios de la teoría del federalismo fiscal (Weingast, 2014); la primera generación de estudios de la hacienda pública fue liderada por Musgrave quien defiende la 
postura de que las transferencias cumplen una función de mecanismo de redistribución en aras de impulsar la eficiencia del actuar económico que lleva a cabo un planificador central benevolente (Musgrave y Musgrave, 1998).

El postulado que defiende la segunda generación, se centra en que los gobiernos regionales pueden satisfacer las necesidades de bienes públicos con mayor capacidad y eficiencia que los gobiernos centrales idea que se convierte en el eje del federalismo fiscal (Porras y García, 2014). Se sostiene la tesis de que los gobiernos locales son más aptos para ejercer el gasto público que un gobierno central, con lo que se defiende la idea de que es mejor descentralizar los recursos para su aplicación administrativa local.

Existen estudios relativamente recientes que fundamentan esta postura; por ejemplo, uno que argumenta que las transferencias pueden promover la eficiencia en el sector público, puesto que de no existir estas, las brechas entre las capacidades fiscales provocarían una migración de los agentes económicos que buscasen una gama de beneficios derivados del gasto público de las regiones más fuertes (Sharma, 2012).

Trabajos académicos contemporáneos sobre federalismo fiscal, defienden que deben de otorgarse más recursos a los gobiernos locales y haciendo una fuerte crítica al centralismo desmesurado en países como México. Las pruebas llevadas a cabo en varios países del orbe, revelan que el gasto ejercido por gobiernos subnacionales depende de los dineros condicionados que envían los gobiernos centrales y cuyas relaciones institucionales y jurídicas, en el caso mexicano al menos, han incrementado el grado de dependencia económica a estas transferencias.

Carmona y Caamal (2018), muestran un estudio en el que los resultados arrojan evidencia de que el sistema de transferencias, concretamente los recursos etiquetados dentro del federalismo fiscal mexicano, contribuyen a reducir la desigualdad salarial cuya incidencia se manifiesta más en municipios que en Estado, argumento que respalda la descentralización fiscal.

Para que la descentralización del gasto público tenga impacto en una mejor distribución de los ingresos debe haber, entre otros aspectos, una relación de gasto descentralizado con una política similar en lo que se refiere a ingresos (Goerl y Seigerling, 2014). Esta postura es respaldada por un estudio hecho entre varios países de la OCDE donde se demuestra una tenue relación entre la descentralización del gasto y la inequidad de los ingresos, encontrándose que los menos favorecidos son aquellos sujetos (naciones) con menores ingresos, pero un mayor impacto en los de ingresos medios (Stossberg, Bartolini y Blöchliger, 2016).

Por otra parte, hay investigaciones que hacen fuerte crítica a externalidades negativas que generan las transferencias de recursos de parte de las federaciones hacía las administraciones públicas locales o regionales. El planteamiento de una dependencia menor de recursos centrales, puede llegar a promover eficiencia en la forma de administrar recursos públicos, atender responsabilidades fiscales y una mayor rendición de cuentas de los gobiernos subnacionales (Ibarra, 2013). Empero, no hay explicaciones de como poder llegar a lograr tal situación, en especial el rubro de la entrega de cuentas claras; ¿quizás la oferta de bienes y servicios públicos que brindan los ayuntamientos mejoran al darles más dinero?, ¿mayor disponibilidad de recursos propios significa finanzas públicas más fuertes? Parecería que al disponer de más recursos hay tendencia a que el gasto crezca aún más incrementando el déficit.

Estos déficits fiscales en las administraciones locales, se pueden entender como un motor de crecimiento puesto que se esperaría que dichos recursos se destinen a gasto en inversión que genere crecimiento económico y por ende aumente la capacidad de recaudación en el futuro; sin embargo, no hay evidencia que sustente que el incremento en deuda tenga como destino inversión pública que sea productiva (García, 2016).

En ese sentido, juega un papel fundamental el tema de los controles jurídico- 
Herrera Díaz de León, Luis Lenin; González Acolt, Roberto; Alcantar López, Cristian Omar y Govea Franco, Ángeles Montserrat

Impacto de las transferencias federales en la deuda municipal de los ayuntamientos mexicanos

administrativos en los efectos que pueda tener la deuda municipal; en un estudio reciente se encontró un porcentaje mayor de deuda en gobiernos subnacionales donde la ley no obligaba a transparentar el nivel de endeudamiento; caso contrario, la deuda fue menor en aquellas entidades que tenían legislaciones que imponían limites definidos a la deuda (Astudillo, et al., 2017).

Bajo los argumentos de estos estudios, la carencia de recursos transferidos por un gobierno federal provocaría en administraciones de menor jerarquía un desbalance presupuestal, dada la obligatoriedad de brindar ciertos bienes públicos y su efectos esperados pero con ausencia de los recursos para hacerlo, ante lo cual habría que plantearse el escenario que los gobiernos subnacionales tendrían que ayudarse de la deuda para poder atender su situación deficitaria; por lo tanto, al transferir más recursos a municipios, estos reportarían menor deuda.

Este trabajo de investigación está enfocado a encontrar elementos que demuestren que a pesar de haber tenido incrementos significativos en los montos de recursos que le ha transferido la federación a los gobiernos municipales dentro del federalismo fiscal mexicano, se ha generado una externalidad negativa que afecta la salud de las finanzas municipales al mostrar incrementos en los montos a pagar de deuda, mediante un análisis de la correlación existente entre transferencias federales y esta última.

La aportación del presente escrito se da principalmente en dos rubros: Propone un modelo que prueba la correlación entre las transferencias federales y los montos de deuda de los municipios; y por otra parte, lleva a cabo un estudio del federalismo fiscal a nivel municipal, nivel de gobierno que no ha sido estudiado como ocurre con las entidades federativas de nivel estatal.

\section{Metodología}

Al llevar a cabo estudios sobre finanzas públicas, estos suelen hacerse analizando el comportamiento de las variables a lo largo del tiempo. Los municipios seleccionados para este análisis fueron los más grandes del país, entendiéndose por ello los que mayor número de habitantes tienen en sus demarcaciones durante el último año analizado (2016); asimismo, se debe hacer mención que se excluyen las delegaciones de la ciudad de México, por dos razones: Su densidad poblacional dispara ciertas tendencias y su asignación presupuestal es atípica.

El trabajo de investigación es de corte cuantitativo, correlacional, explicativo y longitudinal. El enfoque es cuantitativo, puesto que se hace uso de variables numéricas y cuantificables que ayudan a generar indicadores de las variables de estudio que conllevan a confirmar los planteamientos apoyados en instrumentos de análisis estadístico.

La técnica empleada es Datos de Panel, con la finalidad de poder llevar a cabo un análisis de los cambios a lo largo del tiempo; se examinan en diferentes años a los sujetos de estudio, es decir, a las entidades más grandes del país, donde se analiza cómo ha cambiado la variable dependiente con respecto a las independientes en diferentes años (el estudio es Longitudinal). Se llevó a cabo una descripción de la relación entre la variable dependiente con otras variables determinantes, encontrando su relación.

El estudio se ha realizado por medio de modelos econométricos sobre una muestra de 44 Municipios a lo largo de 16 años. Se manejó un Panel de datos desbalanceado, puesto que la variable dependiente no presenta información en todos los años a todos los municipios en las bases de datos consultadas. Las pruebas de hipótesis que se realizaron son de una sola cola para ver si existe significancia al $5 \%$ o al $10 \%$ y validar los modelos estadísticos planteados en este artículo.

De igual manera, la técnica de Datos de Panel permite tomar de manera aleatoria una muestra de una población grande en distintos puntos temporales, lo que incluye entre otros aspectos que se excluya la correlación en los términos de error para distintas observaciones 
(Wooldridge, 2018). Además, Gujarati (2006) explica que:

La técnica de Datos de Panel se utiliza para observar los valores de una o más variables durante un determinado periodo; la misma unidad transversal se estudia a lo largo del tiempo, es decir, en este tipo de estudios se presentan la dimensión espacio y tiempo. (p.613)

El tema que se va a desarrollar está formado por las transferencias federales que reciben los municipios más poblados de México acorde a los registros que se encuentran en el Instituto Nacional de Estadística y Geografía (INEGI), así la recolección de datos no requiere de un instrumento del tipo cuestionario o entrevista; la información se obtiene aplicando una investigación documental. Se utiliza la base de datos del Sistema Estatal y Municipal de Base de Datos (SIMBAD) difundida por el
INEGI, la cual contiene la información antes mencionada (INEGI, 2016).

Estas bases de datos tienen la ventaja de ser las más completas en contenido relativo a las finanzas públicas de los gobiernos sub nacionales y permiten detectar tendencias, además de que por provenir de fuentes oficiales, cumplen con las características de confiabilidad y son difícilmente refutables por ser los canales formales del Estado Mexicano.

La información se maneja por años y per cápita a fin de poder ser comparable; asimismo, toda la información es indexada a un año base que es el 2010, aplicando los coeficientes del Indice Nacional de Precios y Cotizaciones (INPC) para poder cumplir con lo dispuesto en este párrafo. En este sentido, en la Tabla 1 se definen las variables que habrán de utilizarse en la estimación del modelo econométrico.

\section{Tabla 1}

Definición de variables

\begin{tabular}{clc} 
Variable & \multicolumn{1}{c}{ Descripción } & Medición \\
\hline TF & $\begin{array}{l}\text { Son los montos de las transferencias federales que se destinan a los municipios } \\
\text { objeto de estudio }\end{array}$ & Pesos / per cápita \\
PD & Pago que hacen los municipios por concepto de deuda & Pesos / per cápita \\
RPP & $\begin{array}{l}\text { Variable dicotómica de control que incorpora el factor político, mide la } \\
\text { relación con el partido político de la federación en turno. Toma valor de 1 si el } \\
\text { municipio es del mismo partido que la federación y cero si no lo es. }\end{array}$ & Binaria \\
\hline
\end{tabular}

Fuente: Elaboración propia, 2020.

Para el estudio de Datos de Panel se realizan adaptaciones de investigaciones de corte longitudinal, de modelos que han estudiado las finanzas públicas y su impacto en ciertas variables a lo largo del tiempo, como el comportamiento de las finanzas regionales y el efecto sobre el gasto y la inversión de cada fuente de financiación (Sánchez, Gutiérrez y Parra, 1994), los esfuerzos fiscales locales ligados a transferencias (Sour, 2008), o la dependencia de la deuda pública con los recursos centrales (Chíguil, 2014). Se propone la siguiente ecuación:

\section{$P D_{i t}=B_{0}+B_{1} T F_{i t}+B 2 \delta_{0}+u_{i t}$}

Donde:

PDit: Se refiere al pago de la deuda per cápita en los municipios (i) en el año correspondiente $(\mathrm{t})$. 
Herrera Díaz de León, Luis Lenin; González Acolt, Roberto; Alcantar López, Cristian Omar y Govea Franco, Ángeles Montserrat

Impacto de las transferencias federales en la deuda municipal de los ayuntamientos mexicanos

B: Las betas son los parámetros de la ecuación.

TFit: Es el monto de transferencias federales a los municipios per cápita (i) en el año correspondiente $(\mathrm{t})$.

$\delta_{0}$ : Variable dicotómica que indica si hay relación política entre el gobierno municipal y el federal cuando ambos pertenecen al mismo partido político.

$u_{i t}$ : Abarca los valores No observables cambiantes en el tiempo.

La variable dependiente, mide la proporción entre el pago de deuda per cápita y el monto de gasto total por habitante (ver Tabla 1). Por otra parte, la variable independiente, es el monto de las transferencias federales per cápita que envía la federación a los municipios estudiados. Igualmente, se agrega una variable de control dicotómica que ayuda a identificar si el factor político influye al momento de asignar recursos federales a los gobiernos subnacionales; y la forma de medirlo es si el municipio pertenece al mismo partido que el gobierno federal en turno, adquiere valores de 1 en caso de darse esta situación y cero en caso contrario.

El modelo se aplicó con el paquete estadístico e views para poder llevar a cabo el análisis por medio de Datos de Panel, lo que arroja los elementos necesarios para corroborar los planteamientos que se han descrito en este trabajo. Este se corre en tres espacios temporales diferentes: El primero, abarca la totalidad del periodo de tiempo en que se hace el análisis, es decir del año 2000 al 2016 para ver una correlación general; el segundo, engloba los años 2000 a 2007, con el fin de hacer las observaciones antes de la reforma a las reglas de distribución de los recursos federales llevadas a cabo en 2007 y determinar si antes de esta modificación legislativa existía relación entre las variables estudiadas; y el último, hace lo propio para los años posteriores a la reforma citada, es decir, de los años 2008 a 2016 con la finalidad de determinar si se logró cambiar tendencia en el vínculo de las transferencias con pagos de deuda.

De igual forma, el modelo se corre con la estimación de Efectos fijos de sección cruzada y de periodo por default, y con los primeros resultados se hace una prueba de efectos fijos para ver si estos son redundantes, en caso de serlos se corre el modelo nuevamente, pero aplicando estimación de efectos aleatorios y se utiliza la prueba de Hausman, para justificar el uso de este último o no.

En las series de tiempo es muy común que se presente un problema de heterocedasticidad, por lo que se vuelve a estimar el modelo empleando los errores estándar robustos a la heterocedasticidad y a la correlación serial con el método de White para corregir dicho problema (Wooldridge, 2018), lo cual se justifica al ser mayor el número de $\mathrm{N}$ (municipios) que el de años.

La aplicación del método usando el paquete estadístico ahorra tener que llevar ciertas pruebas y en su caso ajustes a los modelos para los problemas de heterocedasticidad y correlación serial sin hacer cambios a coeficientes e indicadores estadísticos, solamente se presentan cambios en la significancia estadística. En ese sentido, Panel de datos, es el método de análisis estadístico longitudinal, que permite hacer posible un análisis muy completo de la relación que guardan las transferencias de recursos que hace la federación en México a los municipios más grandes del país a lo largo de 16 años.

\section{Resultados y discusión}

En este apartado se muestran los resultados de los análisis descritos en la parte metodológica, donde se puede observar la relación que guardan las transferencias federales con los pagos de deuda per cápita de los municipios objeto de estudio de este trabajo de investigación.

El objetivo del análisis llevado a cabo es demostrar si existe una relación positiva entre el pago de la deuda de los 44 municipios más grande del país con los recursos que reciben de la federación por habitante, es decir, si al recibir más transferencias per cápita, se incrementa la deuda por ciudadano de esas entidades 
federales. La Tabla 2, muestra los resultados del modelo ejecutado con datos del periodo que abarca del año 2000 a 2016, empleando el modelo de efectos fijos, aplicando los errores estándar robustos a la heterocedasticidad y a la correlación serial con el método de White.

Tabla 2

Impacto en pago de deuda periodo 2000-2016

\begin{tabular}{lccc}
\hline \multicolumn{1}{c}{ Variable } & Coeficiente & Estadístico t & Probabilidad \\
\hline C & -534.3384 & -1.207738 & 0.2276 \\
\hline Log (transf PC) & 95.34020 & 1.547226 & 0.1223 \\
\hline Factor Político & -12.06485 & -0.540457 & 0.5891 \\
\hline
\end{tabular}

Fuente: Elaboración propia, 2020.

Los resultados que se presentan al aplicar el método de White ajustan los valores $\mathrm{t}$ estadísticos para la constante, las transferencias y el factor político con valores de $-1.207,1.54$ y -0.54 cada uno. El valor de la constante, no es significativo; mientras que en el caso de las transferencias federales, el valor $\mathrm{t}$ es significativo con prueba de un solo lado; $\mathrm{y}$ el factor político, no presenta evidencia de ser estadísticamente significativo.

Se revela una relación positiva entre el pago de deuda de los municipios (por habitante) y las transferencias per cápita que reciben estos, donde el modelo establece que por cada mil pesos que se incrementan los recursos federales por ciudadano, la del pago de deuda por cabeza se incrementa en 95.34 pesos.

Al analizar el segundo bloque temporal de los años 2000 a 2007, se obtienen los siguientes resultados al correr el modelo con el método de efectos fijos, aplicando los errores estándar robustos a la heterocedasticidad y a la correlación serial con el método de White, que se observan en la Tabla 3.

Tabla 3

Impacto en pago de deuda periodo 2000-2007

\begin{tabular}{lccc}
\multicolumn{1}{c}{ Variable } & Coeficiente & Estadístico t & Probabilidad \\
\hline C & -684.8363 & -0.676877 & 0.4991 \\
\hline Log (transf PC) & 116.3348 & 0.813480 & 0.4167 \\
\hline Factor Político & -8.156675 & -0.279345 & 0.7802 \\
\hline
\end{tabular}

Fuente: Elaboración propia, 2020. 
Herrera Díaz de León, Luis Lenin; González Acolt, Roberto; Alcantar López, Cristian Omar y Govea Franco, Ángeles Montserrat

Impacto de las transferencias federales en la deuda municipal de los ayuntamientos mexicanos

Los datos ajustados por el método de White presentan valores $\mathrm{t}$ estadísticos para la constante de -0.676 , las transferencias federales per cápita de 0.813 y factor político de -0.279 . De esta manera, el modelo no tiene validez para este periodo de tiempo analizado al no ser significativo ninguno de los elementos de la ecuación.

El tercer bloque temporal analizado muestra los resultados del Panel de Datos desbalanceado después de los cambios a las reglas de transferencias federales empleando el método de efectos fijos, con el uso de $e$ views y que se muestran en la Tabla 4.

\section{Tabla 4}

Impacto en pago de deuda periodo 2008 a 2016

\begin{tabular}{lccc}
\hline Variable & Coeficiente & Estadístico t & Probabilidad \\
\hline C & -478.3573 & -0.980519 & 0.3276 \\
\hline Log (transf PC) & 86.49963 & 1.313552 & 0.1899 \\
\hline Factor Político & 10.02659 & 0.382699 & 0.7022 \\
\hline
\end{tabular}

Fuente: Elaboración propia, 2020.

Con los números estadísticos y los ajustes con el método de White se exhiben valores para los elementos del modelo planteado, obteniendo un valor de -0.9805 para la constante, 1.31 para las transferencias y 0.3826 para el factor político; por lo que el modelo tiene validez al exponer una relación positiva entre el pago de deuda $\mathrm{y}$ las transferencias per cápita con un nivel de significancia del $10 \%$ al valor de 1.31 , así por cada mil pesos de incrementos en transferencias por habitantes la deuda per cápita se incrementa en 86.49 en este periodo de tiempo. A continuación, se presenta la Tabla 5 con un resumen de los estadísticos y coeficientes de los periodos analizados.

Tabla 5

Resumen de validez del modelo en los periodos analizados

\begin{tabular}{|c|c|c|c|c|c|c|}
\hline \multirow{2}{*}{$\begin{array}{l}\text { Periodos } \\
\text { Variable }\end{array}$} & \multicolumn{2}{|c|}{$2000-2016$} & \multicolumn{2}{|c|}{ 2000-2007 } & \multicolumn{2}{|c|}{ 2008-2016 } \\
\hline & $\underline{\text { Coeficiente }}$ & Estadístico t & $\underline{\text { Coeficiente }}$ & Estadístico t & $\underline{\text { Coeficiente }}$ & Estadístico $\mathrm{t}$ \\
\hline $\mathrm{C}$ & -534.3384 & -1.207738 & -684.8363 & -0.676877 & -478.3573 & -0.980519 \\
\hline $\log (\operatorname{tran} P C)$ & 95.34020 & 1.547226 & 116.3348 & 0.813480 & 86.49963 & 1.313552 \\
\hline Factor Político & -12.06485 & -0.540457 & -8.156675 & -0.279345 & 10.02659 & 0.382699 \\
\hline
\end{tabular}

Fuente: Elaboración propia, 2020. 
Como se puede observar en la Tabla 5, durante todo el periodo analizado de 2000 a 2016, se encuentra evidencia de que hay una relación positiva entre las transferencias federales hacía los municipios más grandes en población de México, el valor estadístico $t$ de 1.54 es superior al valor crítico de 1.2816 para un nivel de significancia del 10\%. Esta situación es diferente para el periodo 2000 a 2007, donde el valor t es inferior al valor crítico y por ende no es significativo ni hay evidencia de que los montos de pago de deuda se vean influenciados por los recursos enviados por la federación.

Finalmente, en el lapso de tiempo que abarca los años posteriores a la reforma de la Ley de coordinación fiscal mexicana, del año 2008 a 2016, los resultados arrojan evidencia de que si hay influencia de los recursos transferidos hacía los montos de pago de deuda de los ayuntamientos analizados, dado que el valor t estadístico tiene un valor de 1.3135 , superior al valor crítico.

De igual manera, se evidencia que en ninguno de los tres periodos de tiempo que se han estudiado el factor político tiene influencia en los pagos de deuda per cápita de los gobiernos subnacionales objeto de estudio de esta investigación, pero hay que mencionar que su papel como variable de control ayuda a que el modelo arroje mejores resultados.

Estos hallazgos demuestran que en general, durante todo el periodo de tiempo que se revisaron las variables establecidas en el modelo, se comprueba la correlación de estas y que esta situación se volvió significativa después de las reformas legislativas hechas a las reglas de operación para transferir recursos a gobiernos de menor nivel jerárquico.

Aunque pequeña, la aportación de los resultados de este trabajo abona elementos a la corriente de pensamiento que toma postura contraria a aquella que sostiene que la descentralización de recursos hacía gobiernos subnacionales debe incrementarse, que caracterizan la segunda generación de estudios de la hacienda pública.

En ese sentido, contribuye el modelo presentado a llevar a cabo críticas que sirvan tanto al gobierno federal mexicano y a sus cámaras legislativas para evaluar si se lograron ciertos objetivos establecidos al momento de hacer las reformas del año 2007 que permitió una mayor transferencia de recursos a los municipios mexicanos.

\section{Conclusiones}

Uno de los temas primordiales dentro del federalismo fiscal y la hacienda pública es la disyuntiva de qué potestades tienen los diferentes niveles de gobierno y con qué recursos debe contar cada uno de ellos. El casi axioma que indica que las administraciones locales pueden atender mejor las necesidades provinciales, ha sido argumento rector para promover la petición de que se transfieran más recursos a estos. Bajo ninguna circunstancia este trabajo pretende diferir de este postulado tan defendido por Murgrave y Oates en sus obras, pero sí se postula el argumento de que mandar más recursos sin tomar en cuenta los efectos negativos que conlleva, no ayuda a la administración pública regional.

Del análisis econométrico llevado a cabo, se demuestra que los gobiernos mientras más recursos reciben de la federación, más se están endeudando, acto contrario a lo que se esperaría que hubiese pasado con las reformas del 2007 en el sistema nacional de transferencias, pues los ayuntamientos mexicanos se endeudaban para cubrir sus déficits y al recibir más dinero vía transferencias menos déficits debería haber y por lo mismo menos deuda.

La falta de mecanismos y regulaciones que se enfoquen al control de deuda tanto a nivel central como en las legislaciones locales que atiendan este tema de manera homogénea, son la principal causa de que se haya llegado a esta tendencia, que es urgente poder corregir en el corto plazo ante la pronta carestía de recursos en la hacienda pública mexicana, derivado de escenarios futuros con precios del petróleo bajos, disminución en la recaudación por la erosión fiscal y disminución de mercados, otrora tiempos fuertes, que suministraban recursos a las arcas federales 
Herrera Díaz de León, Luis Lenin; González Acolt, Roberto; Alcantar López, Cristian Omar y Govea Franco, Ángeles Montserrat

Impacto de las transferencias federales en la deuda municipal de los ayuntamientos mexicanos

vía impuestos.

Algunos municipios abusaron del endeudamiento con bancos privados que de manera irresponsable soltaron recursos a gobiernos trienales a sabiendas que dichos empréstitos iban más allá del periodo de la administración, porque estaban avalados con las transferencias federales que habrían de recibir y que desde 2008 aumentaron su tendencia alcista. Es aquí donde la Secretaría de Hacienda debió haber creado mecanismos para limitar estos excesos, lo cual puede explicar en parte por qué el factor político no influyó en la variable dependiente.

Son realmente pocos los estudios que analizan el tema de los impactos que tienen las transferencias federales en México a un nivel municipal, en parte por la falta de información, y además, porque es más fácil hacer este tipo de estudios a nivel Estatal. Este trabajo explora el menor nivel jerárquico dentro del federalismo fiscal y el impacto que está teniendo en un aspecto tan importante en las finanzas locales como lo es el pago de deuda; sin embargo, es menester hacer mención que se deben hacer trabajos que estudien si estos incrementos en deuda al menos han tenido un impacto positivo en el crecimiento del PIB regional, la calidad de vida de sus habitantes o aumento en infraestructura social.

\section{Referencias Bibliográficas}

Astudillo, M., Blancas, A., y Fonseca, F. C. (2017). La transparencia de la deuda subnacional como mecanismo para limitar su crecimiento. Problemas del Desarrollo, 48(188), 29-54.

Astudillo, M., y Porras, R. (2018). Rendición de cuentas y destino de la deuda pública del Gobierno de la Ciudad de México. Problemas del Desarrollo, 49(194), 3160. $\quad$ https://doi.org/10.22201/ iiec.20078951e.2018.194.62935

Carmona, N., y Caamal, C. G. (2018).
¿Las transferencias federales han logrado reducir la desigualdad en las entidades federativas en México? EconoQuantum, 15(1), 31-51. https:// doi.org/10.18381/eq.v15i1.7111

Chiguil, F. C. (2014). El Sistema Nacional de Coordinación Fiscal (SNCF) y sus efectos en el endeudamiento y el esfuerzo fiscal del Distrito Federal y de las entidades del país, 19952010. Análisis Económico, XXIX(71), 79-111.

García, S. (2016). Federalismo fiscal en México. Porrúa.

Goerl, C-A., y Seiferling, M. (2014). Income inequality, fiscal decentralization and transfer dependency. IMF Working Paper 14/64. International Monetary Fund. https://www.imf.org/external/ pubs/ft/wp/2014/wp1464.pdf

Gujarati, D. N. (2006). Principios de econometría. McGraw-Hill Interamericana.

Ibarra, J. (2013). Entorno político y dependencia financiera de los Estados mexicanos. Gestión y Politica Pública, XXII(1), 3-44.

Instituto Nacional de Estadística y Geografía - INEGI (2016). Síntesis metodológica de la estadística de finanzas públicas estatales $y$ municipales. INEGI. http://internet. contenidos.inegi.org. $\mathrm{mx} /$ contenidos/ Productos/prod serv/contenidos/ espanol/bvinegi/productos/nueva estruc/702825085926.pdf

Machado, G. E. (2017). Gasto público y ciclo electoral en Venezuela. Período 19772012. Revista de Ciencias Sociales (Ve), $\operatorname{XXIII(2),~99-111.~}$

Mandujano, N. (2011). Dependencia e inequidad tributaria de los gobiernos estatales en México. Dimensión Económica, 2(6), 20-36. https:// rde.iiec.unam.mx/revistas/6/ 
articulos/2/21.php

Musgrave, R. A., y Musgrave, P. B. (1998). Hacienda pública: Teórica y aplicada. McGraw-Hill.

Oates, W. E. (1972). Fiscalfederalism. Edward Elgar Publishing.

Pliego, I. H. (2010). El federalismo fiscal en México: Entre la economía y la política. Centro de Estudios Sociales y de Opinión Pública.

Prats, M. A., y Rocamora, A. M. (2018). Consolidación fiscal, un análisis empírico para España. Revista de Ciencias Sociales (Ve), XXIV(1), 9-21.

Ruiz-Porras, A., y García-Vázquez, N. (2014). El federalismo fiscal y las transferencias planeadas hacia los municipios mexicanos: criterios económicos y políticos. Espiral. Estudio sobre Estado y Sociedad, XXI(59), 69-86.

Sánchez, F., Gutiérrez, C., y Parra, J. C. (1994). Transferencias intergubernamentales y comportamiento fiscal de los entes territoriales. Una aproximación econométrica. Coyuntura Económica, XXIV(2), 89-110.

Secretaría de Gobernación - SEGOB (2012). Manual de Transferencias Federales para Municipios. SEGOB, Instituto Nacional para el Federalismo y el Desarrollo Municial (INAFED). http://www.inafed.gob.mx/es/
inafed/Manual de Transferencias Federales para Municipios

Sharma, C. K. (2012). Beyond gaps and imbalances: Re $\square$ structuring the debate on intergovernmental fiscal relations. Public Administration, 90(1), 99128. https://doi.org/10.1111/j.14679299.2011.01947.x

Sour, L. (2008). Un repaso de los conceptos sobre capacidad y esfuerzo fiscal, y su aplicación en los gobiernos locales mexicanos. Estudios Demográficos $y$ Urbanos, 23(2), 271-297. http:// dx.doi.org/10.24201/edu.v23i2.1312

Stiglitz, J. E., y Rosengard, J. K. (2016). La economía del sector público. Antoni Bosch Editor.

Stossberg, S., Bartolini, D., y Blöchliger, H. (2016). Fiscal decentralization and income inequality: Empirical evidence from OECD Countries. OECD Economics Department Working Papers, (1331), OECD Publishing. https://doi. org/10.1787/5jlpq7tm05r6-en.

Weingast, B. R. (2014). Second generation fiscal federalism: Political aspects of decentralization and economic development. World Development, 53, 14-25. https://doi.org/10.1016/j. worlddev.2013.01.003.

Wooldridge, J. M. (2018). Introducción a la econometría. Cengage Learning. 